\title{
Derivation of Line Shape Function in the Optical Conductivity by a New Diagram Method
}

\author{
Nam Lyong Kang ${ }^{1}$ and Sang Don $\mathrm{Choi}^{2}$ \\ ${ }^{1}$ Department of Applied Nanoscience, Pusan National University, Miryang 627-706, Republic of Korea \\ ${ }^{2}$ Department of Physics, Kyungpook National University, Daegu 702-701, Republic of Korea \\ Correspondence should be addressed to Nam Lyong Kang; nlkang@pusan.ac.kr
}

Received 11 February 2014; Revised 22 April 2014; Accepted 6 May 2014; Published 25 May 2014

Academic Editor: Jörg Fink

Copyright (C) 2014 N. L. Kang and S. D. Choi. This is an open access article distributed under the Creative Commons Attribution License, which permits unrestricted use, distribution, and reproduction in any medium, provided the original work is properly cited.

A new diagram method for the line shape function in the optical conductivity formula is introduced and the result obtained applying the method to an electron-phonon system is compared with that derived using the projection-reduction method. The result satisfies the population criterion, which states that the distribution functions for electrons and phonons should be combined in multiplicative forms and gives physical intuition to quantum dynamics of electrons in a solid. This method can be called the "KC diagram" method because it originates from the proper application of the Kang-Choi reduction identity and a state-dependent projection operator.

\section{Introduction}

Studies of the optical transitions in electron systems are powerful for examining the electronic properties of solids because the absorption line shapes are quite sensitive to the type of scattering mechanism affecting the transport of electrons and to the interaction of electrons with intense laser light. A perturbation-based study is a general method for gaining knowledge on the dynamics of a system. This consists of dividing the Hamiltonian into an exactly soluble part and nontrivial perturbative part, the effects of which are studied in perturbative order. The Feynman diagram is the most popular method for representing the terms in perturbative expressions. This diagrammatic method can be used directly for reasoning and problem solving as well as for representing the perturbative expressions by drawings. The easily recognizable topology of the diagrams makes the diagrammatic method a powerful tool for constructing approximation schemes. In addition, the diagrammatic representation can be a suggestive tool providing physical intuition vital to quantum dynamics by increasing the diagrams to a representation for possible alternative physical processes.

The standard diagram method can represent the trajectory of the particles well in the intermediate states of the scattering processes. On the other hand, when the method is applied to obtaining the line shape (or self-energy) function for the electron-phonon system, the Fermi distribution functions for the electrons and the Bose distribution functions for the phonons are simply added [1-11], which violates the "population criterion" suggesting that the Fermi and Bose distribution functions for electrons and phonons should be combined in multiplicative forms. In other words, the theory can be said to be suitable if the Fermi distributions are multiplied by the Bose distributions in the formalism because the electrons (fermions) and phonons (bosons) belong to different categories in quantum-statistical physics. In spintronics, which has attracted considerable attention in recent years, preserving the information injected into spin over a practical time scale is important for the spintronics devices. Therefore, it is crucial to understand how the distribution functions are included because the temperature dependence of the spin relaxation time might be caused by the Bose and Fermi distribution functions [8-12].

On the other hand, although a dynamical conductivity formula for superconducting materials could be obtained from Eliashberg theory [13] where the electron-phonon interaction is described by the spectral function, the result did not contain the distribution function for phonons, so 
the phonon emissions and absorptions as well as photon absorptions and emissions in all electron transition processes could not be presented in an organized manner. Nam [14, 15] derived general expressions for the current density for superconductors in a transverse electromagnetic field from a Green function method but the result could not explain the inverse relaxation time $(2 \Gamma / \hbar)$ from the fully microscopic viewpoint. In the present paper, we derive an explicit form of $\Gamma$ from a new diagram method.

The present authors developed some projection methods for the optical transition formulae for electron-phonon systems and used them to calculate the line widths in semiconductors $[12,16-18]$. Normally, the resolvent factor included in the conductivity tensor is expanded using projectors, and a perturbative range of formulae can be obtained. Recently, the formalism was improved by introducing nonlinear terms near the resonance points, yielding a meaningful result including the Fermi and Bose distribution functions properly with the proper use of the projection-reduction (PR) method combining the Kang-Choi reduction identity (KCRI) and state-dependent projection operator (SDPO) [18].

This paper introduces a new diagram method using the vertex linkers called "springs" and "loops" for the line shape function in the optical conductivity tensor and compares the result obtained applying the method to an electron-phonon system with that derived using the PR method. We further show that physical intuition vital to the quantum dynamics of electrons in a solid can be obtained by the diagrams.

\section{Method}

When an electromagnetic wave of frequency $\omega$ is applied to a system along the $l(=x, y, z)$ direction, the linear optical conductivity for a system of electrons interacting with phonons can be derived using the state-dependent projection method as follows [18]:

$$
\sigma_{k l}(\omega)=e \sum_{\alpha, \beta} \frac{\left(r_{l}\right)_{\alpha \beta}\left(j_{k}\right)_{\beta \alpha}\left(f_{\alpha}-f_{\beta}\right)}{\hbar \omega+E_{\alpha \beta}-\Gamma_{\alpha \beta}(\omega)},
$$

where $(X)_{\alpha \beta} \equiv\langle\alpha|X| \beta\rangle$ for electron states $\alpha$ and $\beta, r_{l}$ is the $l$ component of the electron position vector, $j_{k}$ is the $k$ component of the single electron current density operator, $f_{\alpha}$ is the Fermi distribution function for an electron with energy $E_{\alpha}$, and $E_{\alpha \beta} \equiv E_{\alpha}-E_{\beta}$. The denominator in (1) represents the transition from the initial state $\alpha$ to the final state $\beta$ with a photon absorption of frequency $\omega$. If there is no phonon scattering, the line shape would be like a delta function. However, as the electrons are scattered by phonons, the shape is broadened, so the line shape function, $\Gamma_{\alpha \beta}(\omega)$, is involved, which is derived by a diagram method. When an electron-phonon interaction is involved in an electron transition, the electron undergoes an implicit transition from an implicit state, which is coupled with the initial (final) state by an electron-phonon interaction, to the final (initial) state with phonon absorption (or emission) and photon emission (or absorption). The implicit transition forms a loop because the phonon absorption process maintains a balance with the

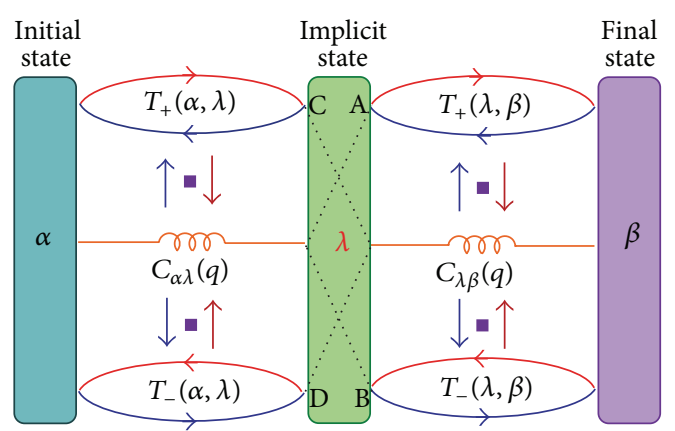

FIGURE 1: All possible electron transition processes in the line shape function, $\Gamma_{\alpha \beta}(\omega)$, in (1). The springs and loops correspond to the interaction coupling factors, $C_{\alpha \beta}(q)$, and the implicit transition factors, $T_{ \pm}(\alpha, \beta)$, respectively. $A, B, C$, and $D$ are the same as those in (6); for example, process A consists of the left spring and right upper loop.

phonon emission process and there are reverse processes because phonon (photon) absorption and emission processes occur independently.

The rules for deriving the line shape function in the optical conductivity tensor by the diagram method are as follows.

Rule 1. Implicit states induced by the electron-phonon interaction exist between the initial and final states.

Rule 2. An implicit state is connected to the initial (or final) state by a proper interaction coupling factor ( $C$-factor), $C_{\alpha \beta}(q)$, which is represented by a spring (see Figure 1):

$$
C_{\alpha \beta}(q) \equiv V_{q}\langle\alpha|\exp (i q \cdot r)| \beta\rangle,
$$

where $V_{q}$ is the coupling factor that depends on the mode of the phonons. A spring emits or absorbs a phonon with wave vector $q$.

Rule 3. There are two implicit transition factors ( $T$-factors) for each $C$-factor. The one forms a clockwise loop, $T_{+}(\alpha, \beta)$, and the other forms a counterclockwise loop, $T_{-}(\alpha, \beta)$ (see Figure 1):

$$
T_{ \pm}(\alpha, \beta) \equiv G_{\alpha \beta}\left( \pm \omega_{q}\right) P_{ \pm}(\alpha, \beta) .
$$

Here the $G$-factor, $G_{\alpha \beta}\left( \pm \omega_{q}\right)$, is defined as

$$
G_{\alpha \beta}\left( \pm \omega_{q}\right) \equiv \delta\left(\hbar \omega+E_{\alpha}-E_{\beta} \mp \hbar \omega_{q}\right)
$$

by which the energy conservation is satisfied; that is, $E_{\beta}=$ $E_{\alpha}+\hbar \omega \mp \hbar \omega_{q}$ and the population factors, $P_{ \pm}(\alpha, \beta)$, are defined as

$$
\begin{aligned}
& P_{+}(\alpha, \beta) \equiv\left(N_{q}+1\right) f_{\alpha}\left(1-f_{\beta}\right)-N_{q} f_{\beta}\left(1-f_{\alpha}\right), \\
& P_{-}(\alpha, \beta) \equiv N_{q} f_{\alpha}\left(1-f_{\beta}\right)-\left(N_{q}+1\right) f_{\beta}\left(1-f_{\alpha}\right),
\end{aligned}
$$

where $N_{q}$ is the Bose distribution function for phonons with energy $\hbar \omega_{q}$. In a loop, the red (upper) and blue (lower) half circles correspond to phonon emission $\left(N_{q}+1\right)$ and phonon absorption $\left(N_{q}\right)$, respectively. 
Rule 4. A $C$-factor multiplied by a $T$-factor becomes an element in the line shape function.

Rule 5. Finally, summing the elements, after summing each element over all the phonon wave vectors and implicit states, we obtain the line shape function.

\section{Results}

Using the rules, the line shape function can be obtained. There are four elements (Figure 1). The first two terms correspond to $C_{\alpha \lambda}(q)$ and the other two terms correspond to $C_{\lambda \beta}(q)$, where $\lambda$ denotes the implicit state. The springs emit or absorb a phonon (purple square) with a wave vector $q$, through which the implicit states are created from the initial or final states. The blue vertical arrows pointing to the loop mean that a phonon emitted from the spring is absorbed by an electron during the blue implicit transition, and the red vertical arrows pointing to the spring mean that a phonon emitted by an electron during the red implicit transition is absorbed by the spring. A phonon created by a left spring can be absorbed by an electron during the transitions between the initial $(\alpha)$ and implicit $(\lambda)$ states of the left blue (lower) half circles or the left spring can absorb a phonon emitted by an electron during the transitions of the left red (upper) half circles. The right red (blue) half circles mean that the implicit transitions between the implicit state and the final state $(\beta)$ occur emitting (absorbing) a phonon to (created from) the right spring. A photon is absorbed in the forward (rightward) process and emitted in the backward (leftward) process.

From Rule 5, the result is given as

$$
\begin{aligned}
& \Gamma_{\alpha \beta}(\omega)\left(f_{\alpha}-f_{\beta}\right) \\
&=\sum_{q, \lambda}\left\{\left|C_{\alpha \lambda}(q)\right|^{2}\left[G_{\lambda \beta}\left(+\omega_{q}\right) P_{+}(\lambda, \beta)+G_{\lambda \beta}\left(-\omega_{q}\right) P_{-}(\lambda, \beta)\right]\right. \\
&+\left[G_{\alpha \lambda}\left(+\omega_{q}\right) P_{+}(\alpha, \lambda)+G_{\alpha \lambda}\left(-\omega_{q}\right) P_{-}(\alpha, \lambda)\right] \\
&\left.\quad \times\left|C_{\lambda \beta}(q)\right|^{2}\right\}=\mathrm{A}+\mathrm{B}+\mathrm{C}+\mathrm{D} .
\end{aligned}
$$

The physical meaning of the first term (A) in (6) is as follows: $G_{\lambda \beta}\left(+\omega_{q}\right)$ means that the energy of an implicit state $\lambda$ is determined by the energy of the final state $\beta$, photon energy, and phonon energy, so that energy conservation can be satisfied; that is, $E_{\lambda}+\hbar \omega=E_{\beta}+\hbar \omega_{q} \cdot\left|C_{\alpha \lambda}(q)\right|^{2}$ means that the implicit state $\lambda$ is coupled with the initial state $\alpha$ by a phonon with a wave vector $q . P_{+}(\lambda, \beta)$ means that the reverse implicit transition from the final state $\beta$ to the implicit state $\lambda$ with phonon absorption should be subtracted from the forward implicit transition from the implicit state to the final state with phonon emission. This means that when an electron-phonon interaction is involved in an electron transition, a transition occurs via implicit states induced by local fluctuations. A net transition is possible because the implicit states are determined by the energies of the final (or initial) state, photon, and phonon according to (4). The other three terms in (6) can be interpreted in a similar manner.

Although $\Gamma_{\alpha \beta}(\omega)$ is called the line shape function, the line width (or relaxation rate) must be calculated from plotting (1) against the frequency considering (6). The line width, however, can be obtained directly from (6) if the Lorentzian approximation is assumed for the weak scattering. Therefore, all the states given by $\lambda$ are called the implicit states because they are included only in $\Gamma_{\alpha \beta}(\omega)$. The implicit states are determined by the delta functions for energy conservation given in (4) and momentum conservation, which is given by (2). The transition from the initial state $\alpha$ to the final state $\beta$ occurs via two implicit transitions, $T_{ \pm}(\alpha, \lambda)$ and $T_{ \pm}(\lambda, \beta)$, and the implicit state is connected to the initial or final states by $C_{\alpha \lambda}(q)$ or $C_{\lambda \beta}(q)$. Although the implicit transitions are not measured directly, they should be considered in the calculations. Note that the other theories [1-11] cannot provide any diagrammatic representation because they contain the sums of two distribution functions, such as $\left(N_{q}+1 / 2 \pm 1 / 2 \mp f_{\alpha}\right)$.

Figure 2 shows all the possible implicit transition processes in Figure 1. The loops (1), (2), (3), and (4) correspond to $\mathrm{C}, \mathrm{A}, \mathrm{D}$, and $\mathrm{B}$ in (6), respectively. The blue triangle, green circle, and purple square denote the photon, electron, and phonon, respectively. The meaning of stage (a) is as follows. The electrons in the initial state of loop (1) and implicit state of loop (2) emit phonons to the springs and absorb photons. Two springs emit phonons to loops (3) and (4), and the electrons in the initial state of loop (3) and the implicit state of loop (4) absorb photons. (b) and (e) are virtual stages. Stage (c) means that the electrons in the initial state of loop (1) and implicit state of loop (2) transit to the implicit and final states by emitting phonons to the springs and absorbing photons, and the electrons in the initial state of loop (3) and the implicit state of loop (4) transit to the implicit and final states by absorbing phonons emitted from the springs and absorbing photons. Stage (c) becomes stage (f) if the electrons in the implicit state of loop (1) and final state of loop (2) absorb phonons from the springs and emit photons, and the electrons in the implicit state of loop (3) and final state of loop (4) emit phonons to the springs and emit photons.

\section{Conclusions}

So far, we introduced a new diagram method for the line shape function in the optical conductivity tensor for an electron-phonon system. We showed that the same result as that derived using the PR method could be obtained more easily and in a physically acceptable manner using the present diagram method. The phonon emissions and absorptions as well as the photon emissions and absorptions in all electron transition processes can be analyzed in an organized manner because the line shape functions include the electron and phonon distribution functions properly. These diagrams should not be confused with the time-ordered diagrams in the Feynman scheme [19] or with the temperature diagrams in the Feynman-like scheme $[20,21]$ because the present diagram method is not the one representing the trajectories of the particles in the intermediate stages of the scattering 


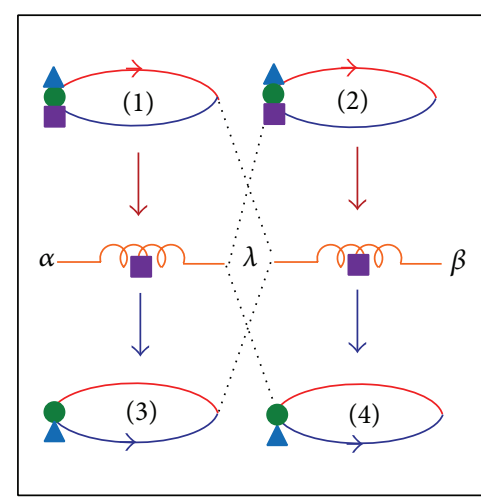

(a)

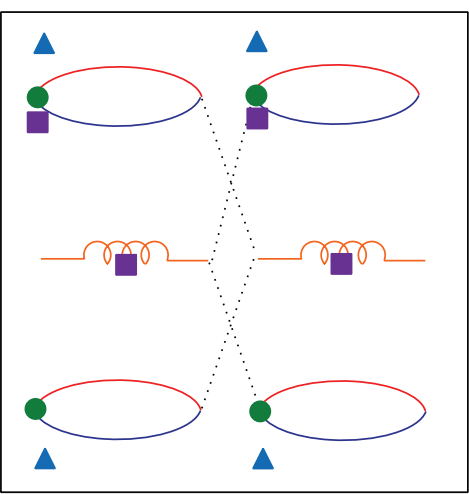

(f)

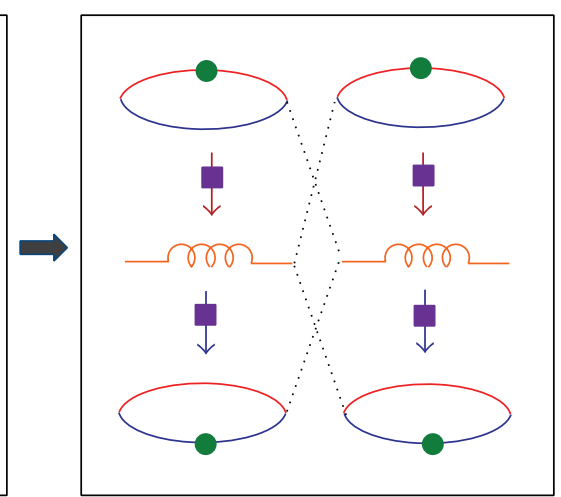

(b)

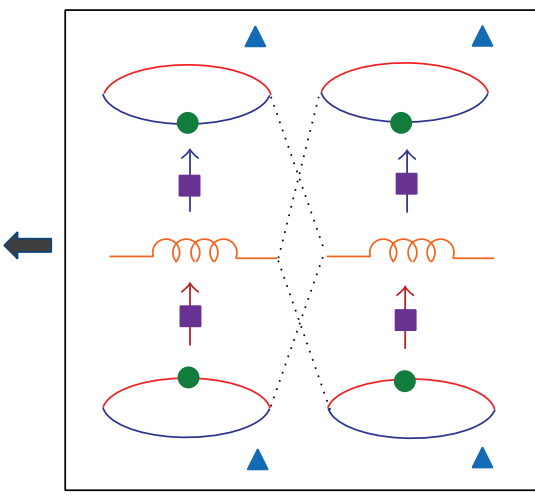

(e)

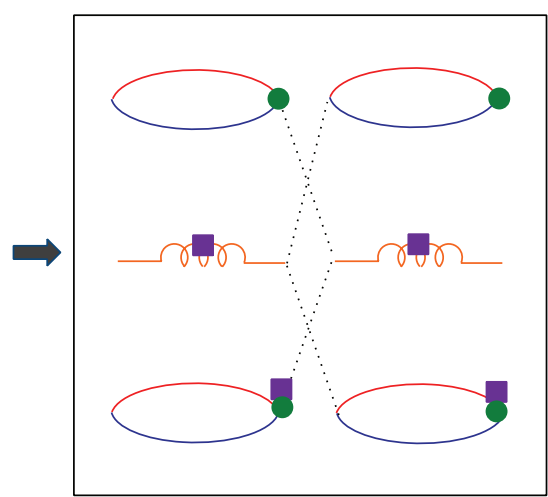

(c)

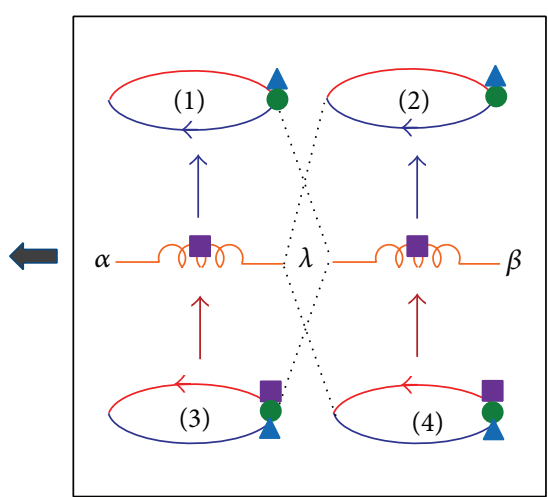

(d)

Figure 2: All the possible implicit transition processes in Figure 1. Loops (1), (2), (3), and (4) correspond to C, A, D, and B in (6), respectively. The blue triangle, green circle, and purple square denote the photon, electron, and phonon, respectively.

processes. On the other hand, this method can be called the "KC diagram" because it is based on the electron-phonon population topology originated by the proper application of KCRI and SPDO. The experimental results could be explained by the formulae considered in this paper $[16,17]$ and physical intuition for the quantum dynamics of an electron in solids could be provided using the present diagrammatic method. Therefore, a proper theory that is physically acceptable and generally applicable can be obtained using the KCRI and SDPO. This method is expected to be applicable further to other electron transition phenomena, which is left for future studies.

\section{Conflict of Interests}

The authors declare that there is no conflict of interests regarding the publication of this paper.

\section{Acknowledgment}

This work was supported by the Pusan National University Research Grant, 2014.

\section{References}

[1] L. Hedin and S. Lundqvist, Solid State Physics, vol. 15, Academic Press, New York, NY, USA, 1969.

[2] G. Grimvall, The Electron-Phonon Interaction in Metals, NorthHolland, New York, NY, USA, 1981.

[3] P.-B. Allen and B. Mitrovvich, Solid State Physics, vol. 37, Academic Press, New York, NY, USA, 1982.

[4] G. D. Mahan, Many-Particle Physics, Plenum Press, New York, NY, USA, 1990.

[5] B. Hellsing, A. Eiguren, F. Reinert et al., "Lifetime of holes and electrons at metal surfaces; electron-phonon coupling," Journal of Electron Spectroscopy and Related Phenomena, vol. 129, no. 23, pp. 97-104, 2003.

[6] F. Giustino, M. L. Cohen, and S. G. Louie, "Small phonon contribution to the photoemission kink in the copper oxide superconductors," Nature, vol. 452, no. 7190, pp. 975-978, 2008.

[7] I. A. Nechaev, I. Y. Sklyadneva, V. M. Silkin, P. M. Echenique, and E. V. Chulkov, "Theoretical study of quasiparticle inelastic lifetimes as applied to aluminum," Physical Review B: Condensed Matter and Materials Physics, vol. 78, no. 8, Article ID 085113, 13 pages, 2008.

[8] B. Huang, D. J. Monsma, and I. Appelbaum, "Coherent spin transport through a 350 micron thick silicon wafer," Physical Review Letters, vol. 99, no. 17, Article ID 177209, 4 pages, 2007. 
[9] J. L. Cheng, M. W. Wu, and J. Fabian, "Theory of the spin relaxation of conduction electrons in silicon," Physical Review Letters, vol. 104, no. 1, Article ID 016601, 4 pages, 2010.

[10] P. Li and H. Dery, "Spin-orbit symmetries of conduction electrons in silicon," Physical Review Letters, vol. 107, no. 10, Article ID 107203, 5 pages, 2011.

[11] O. D. Restrepo and W. Windl, "Full first-principles theory of spin relaxation in group-IV materials," Physical Review Letters, vol. 109, no. 16, Article ID 166604, 5 pages, 2012.

[12] N. L. Kang and S. D. Choi, "Calculation of electron spin relaxation time in germanium using the projection-reduction method," Japanese Journal of Applied Physics, vol. 53, no. 1, Article ID 013001, 4 pages, 2014.

[13] W. Lee, D. Rainer, and W. Zimmermann, "Holstein effect in the far-infrared conductivity of high $T_{c}$ superconductors," Physica C: Superconductivity and Its Applications, vol. 159, no. 5, pp. 535544, 1989.

[14] S. B. Nam, "Theory of electromagnetic properties of superconducting and normal systems. I*," Physical Review, vol. 156, no. 2, pp. 470-486, 1967.

[15] S. B. Nam, "Theory of electromagnetic properties of strongcoupling and impure superconductors. $\mathrm{II}^{*}, "$ Physical Review, vol. 156, no. 2, pp. 487-493, 1967.

[16] N. L. Kang, J. Y. Ryu, and S. D. Choi, "Derivation of linewidths for optical transitions in quantum wells due to longitudinal optical phonon scattering," Journal of Physics Condensed Matter, vol. 14, no. 41, pp. 9733-9742, 2002.

[17] N. L. Kang and S. D. Choi, "Scattering effects of phonons in two polymorphic structures of gallium nitride," Journal of Applied Physics, vol. 106, no. 16, Article ID 063717, 6 pages, 2009.

[18] N. L. Kang and S. D. Choi, "Projection-reduction approach to optical conductivities for an electron-phonon system and their diagram representation," ISRN Condensed Matter Physics, vol. 2014, Article ID 719120, 23 pages, 2014.

[19] R. P. Feynman, "Space-time approach to quantum electrodynamics," Physical Review, vol. 76, no. 6, pp. 769-789, 1949.

[20] A. L. Fetter and J. D. L. Walecka, Quantum Theory of ManyParticle Systems, McGraw-Hill, New York, NY, USA, 1971.

[21] R. D. A. Mattuch, Guide to Feynman Diagrams in the ManyBody Problem, McGraw-Hill, New York, NY, USA, 1976. 

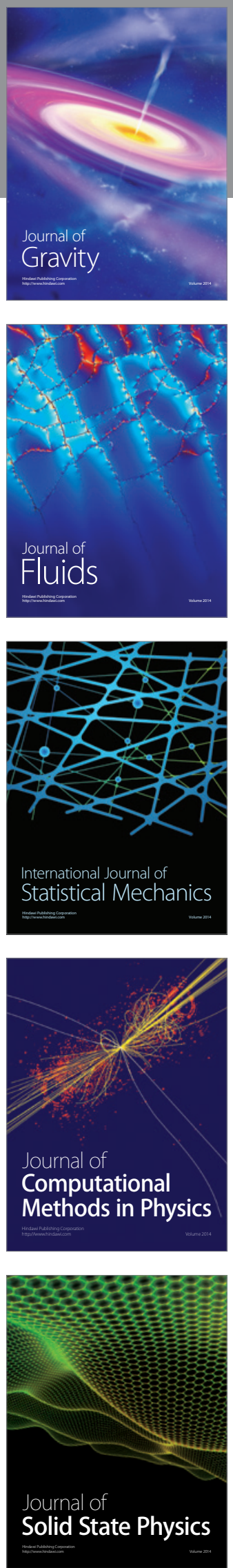

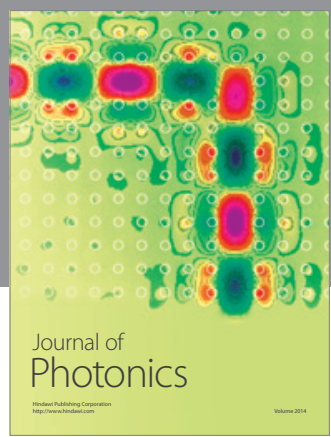

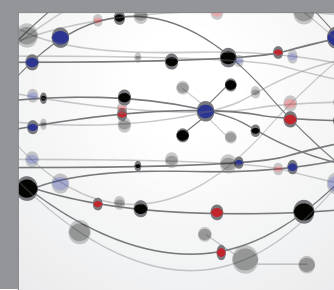

The Scientific World Journal

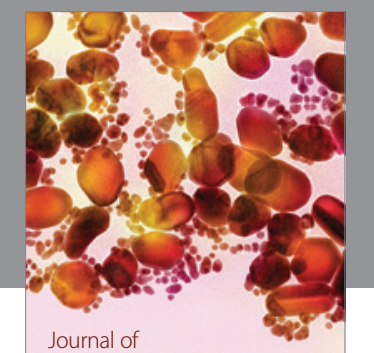

Soft Matter
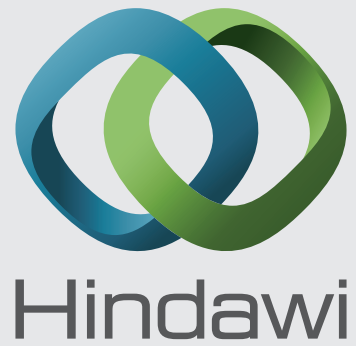

Submit your manuscripts at

http://www.hindawi.com
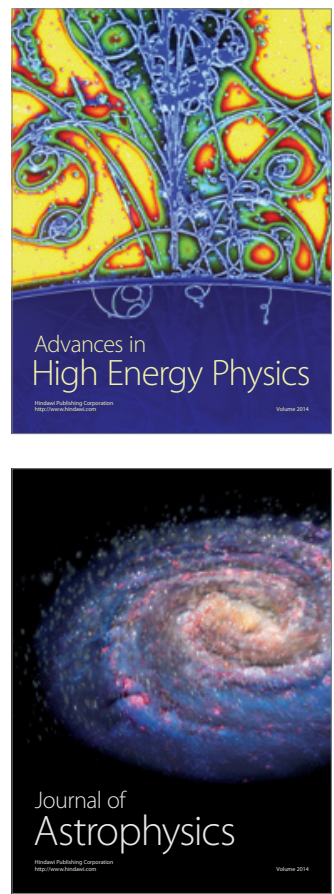
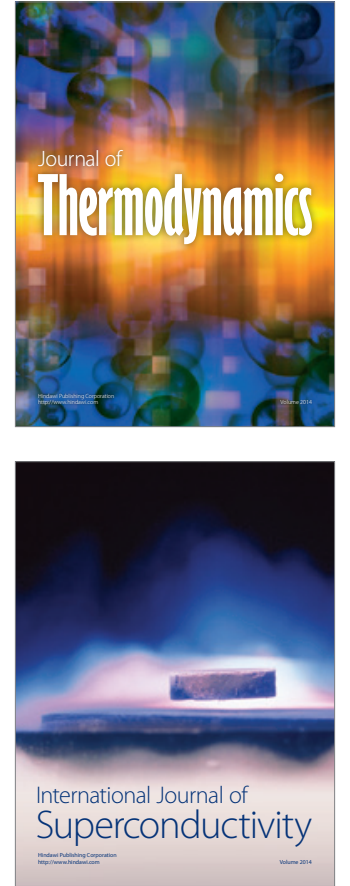
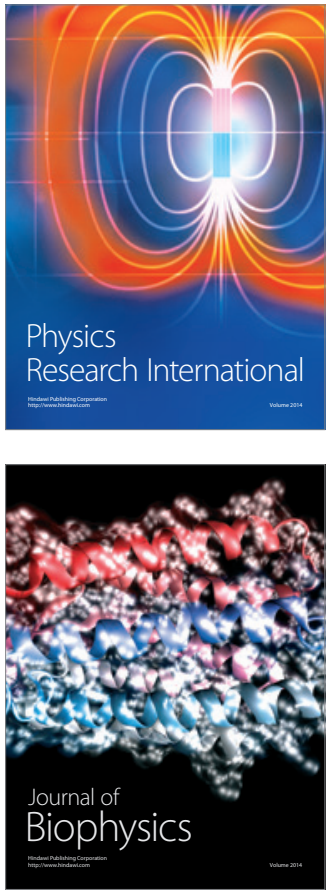
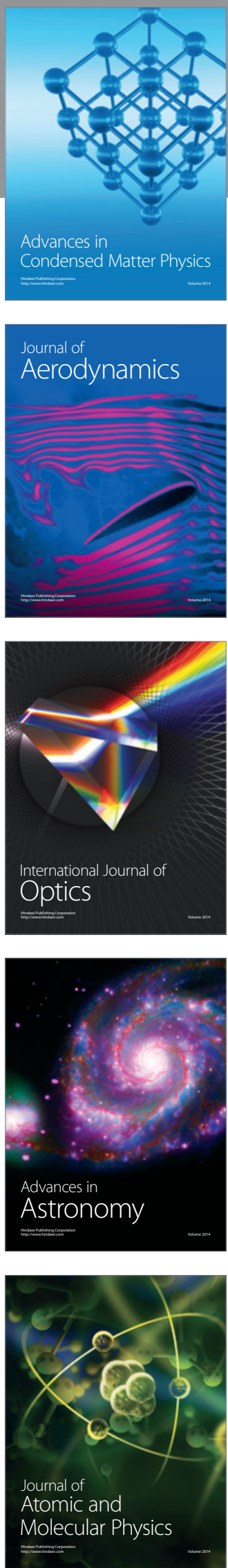